\title{
Replication Specificity Elements of the Worland Strain of Beet Curly Top Virus Are Compatible with Those of the CFH Strain But Not Those of the Cal/Logan Strain
}

\author{
Drake C. Stenger
}

\begin{abstract}
Department of Biological Sciences and Plant Molecular Biology Center, Northern Illinois University, DeKalb 60115.
Current address of D. C. Stenger: Agricultural Research Service, United States Department of Agriculture, and Department of Plant Pathology, University of Nebraska, Lincoln 68583.
\end{abstract}

Accepted for publication 24 July 1998.

\begin{abstract}
Stenger, D. C. 1998. Replication specificity elements of the Worland strain of beet curly top virus are compatible with those of the CFH strain but not those of the Cal/Logan strain. Phytopathology 88:1174-1178.

Cloned genomes of the $\mathrm{CFH}$, Worland, and $\mathrm{Cal} / \mathrm{Logan}$ strains of beet curly top virus (BCTV) served as helper viruses to trans-replicate defective (D) DNAs that are incapable of self-replication due to deletions within the $\mathrm{C} 1$ open reading frame encoding the replication initiator (Rep) protein. The Logan Rep protein could trans-replicate a Logan-derived D DNA in a transient replication assay conducted in Nicotiana benthamiana leaf disks. However, the Logan Rep protein was unable to trans-replicate D DNAs derived from the CFH or Worland strains. In contrast, the Rep proteins of the CFH and Worland strains could trans-replicate CFH or Worland D DNAs, but not a Logan D DNA. These results indicate that the cis- and

trans-acting replication specificity elements of the $\mathrm{CFH}$ and Worland strains are compatible and that the three strains of BCTV may be divided into two groupings based upon replication specificity determinants. A comparison of amino acid sequences of the Rep protein for the three BCTV strains suggests that the trans-acting replication specificity element may reside in one or more of 12 amino acid residues that are identical; in two amino acid residues that are chemically similar among the $\mathrm{CFH}$ and Worland Rep proteins, yet are different in the Logan Rep protein; or in both. Properties including replication specificity, nucleotide sequence identity, and symptom expression were used as criteria to propose separate species designations for each of the three BCTV strains. In this proposal, the Cal/ Logan strain retains the name BCTV, CFH and the closely related Iranian isolate are designated beet severe curly top virus, and Worland is designated beet mild curly top virus.
\end{abstract}

Beet curly top virus (BCTV) is the type member of the genus Curtovirus (formerly subgroup II) in the family Geminiviridae. Three strains (Cal/Logan, CFH, and Worland) of BCTV have been characterized at the molecular level $(1,8,11,12,15,18)$ and differ sufficiently in nucleotide sequence ( 79 to $83 \%$ identity) to be considered distinct species, although separate taxon designations have not been formally adopted (18). While the virion sense genes V1, V2, and V3 are highly conserved (>95\% nucleotide sequence identity) among all characterized strains of BCTV, DNA sequences of the intergenic region containing the origin of DNA replication (ori) and those encoding the complementary sense genes $\mathrm{C} 1, \mathrm{C} 2, \mathrm{C} 3$, and $\mathrm{C} 4$ are more divergent $(1,8,11,12,18)$.

Essential cis- and trans-acting elements conferring strain-specific replication properties have been mapped to portions of the BCTV genome using chimeric viruses derived from the $\mathrm{CFH}$ and $\mathrm{Cal} / \mathrm{Logan}$ strains $(2,3)$. The cis-acting strain-specific determinant of BCTV DNA replication has been fine-mapped to a 13- to 14-nucleotide sequence motif within the ori (3). The homologous element in whitefly-transmitted geminiviruses (genus Begomovirus, formerly subgroup III) is known to play a role in replication specificity (5)

Corresponding author: D. C. Stenger; E-mail address: dstenger@unlinfo.unl.edu

Mention of proprietary or brand names are necessary to report factually on available data; however, the USDA neither guarantees nor warrants the standard of the product, and the use of the name by the USDA implies no approval to the exclusion of others that also may be suitable.

Publication no. P-1998-0827-02R

This article is in the public domain and not copyrightable. It may be freely reprinted with customary crediting of the source. The American Phytopathological Society, 1998. and to bind the cognate geminivirus replication initiator (Rep) protein (4). Wheat dwarf virus (genus Mastrevirus, formerly subgroup I) also has cis-acting elements within the ori that are required for replication and bind Rep protein with high affinity (10). The trans-acting replication specificity element of BCTV has been mapped (2) to the amino-terminal region (Logan amino acid residues 3 to 89 ) of the Rep protein encoded by the $\mathrm{C} 1$ open reading frame (ORF). The trans-acting specificity element of a begomovirus, tomato yellow leaf curl virus, also has been mapped (9) to a similar region of the Rep protein (amino acid residues 1 to 116). However, the precise location of the trans-acting specificity element within the amino-terminal region of the geminivirus Rep protein remains to be determined.

The cis- and trans-acting strain-specific replication elements of the Cal/Logan and CFH strains of BCTV are not separately interchangeable. However, if both cognate elements are exchanged, the resulting chimeric virus self-replicates (2). Chimeric viral genomes bearing heterologous combinations of cis- and trans-acting replication specificity elements derived from the $\mathrm{Cal} / \mathrm{Logan}$ and $\mathrm{CFH}$ strains were self-replication deficient, yet able to complement one another in coinoculation experiments (2). Self-replication competency of these chimeric genomes could be restored by site-directed mutagenesis in which certain nucleotides of the cis-acting specificity element of the ori were altered to those of the cognate virus from which the Rep protein was derived (3). Sequences of the cisacting replication specificity element of the $\mathrm{CFH}$ and Worland strains resemble one another more than they resemble the corresponding element of the Cal/Logan strain (13). The region of the Rep protein containing the trans-acting specificity element is divergent among the three strains. Nonetheless, phylogenetic analysis indicated that the Worland and CFH Rep proteins share a most recent common ancestor (1). 
Field isolates of BCTV often contain less-than-genome-length defective (D) DNA molecules (14) that likely arise through deletion events. All three strains of BCTV readily produce D DNAs de novo upon systemic infection of plants infected using cloned inocula devoid of defective genomes $(6,13,20)$. For all cases in which nucleotide sequences were examined $(6,20)$, BCTV D DNAs were selfreplication defective due to deletions within the $\mathrm{C} 1 \mathrm{ORF}$ encoding the Rep protein, although cis-acting sequences of the ori were retained. When present as tandem repeats chromosomally integrated into transgenic plants, D DNAs of the California (7) or Logan (13) isolates of the $\mathrm{Cal} /$ Logan strain may be mobilized and amplified upon challenge inoculation with the helper virus genome from which they were derived. The concomitant delay and amelioration of symptoms and reduced accumulation of helper virus DNA indicates that these D DNAs interfere with helper virus replication and are, therefore, defective-interfering (DI) DNAs. However, when CFH or Worland were used as helper viruses in challenge inoculations of transgenic plants bearing a Logan-derived DI DNA, no delay or amelioration of symptoms occurred and the Logan DI DNA was not mobilized or amplified (13). These results confirm the incompatibility of the replication specificity elements of Logan and CFH and indicate that the trans-acting replication specificity element of Worland is not compatible with the cis-acting element of Logan. The compatibility of $\mathrm{CFH}$ and Worland replication specificity elements and the compatibility of the Logan trans-acting element with the Worland cis-acting element have not been evaluated. In the experiments reported here, each of the three BCTV strains were used as helper viruses that provided the trans-acting specificity element of the Rep protein and were assessed for their ability to trans-replicate D DNA molecules bearing ori sequences derived from each of the three BCTV strains.

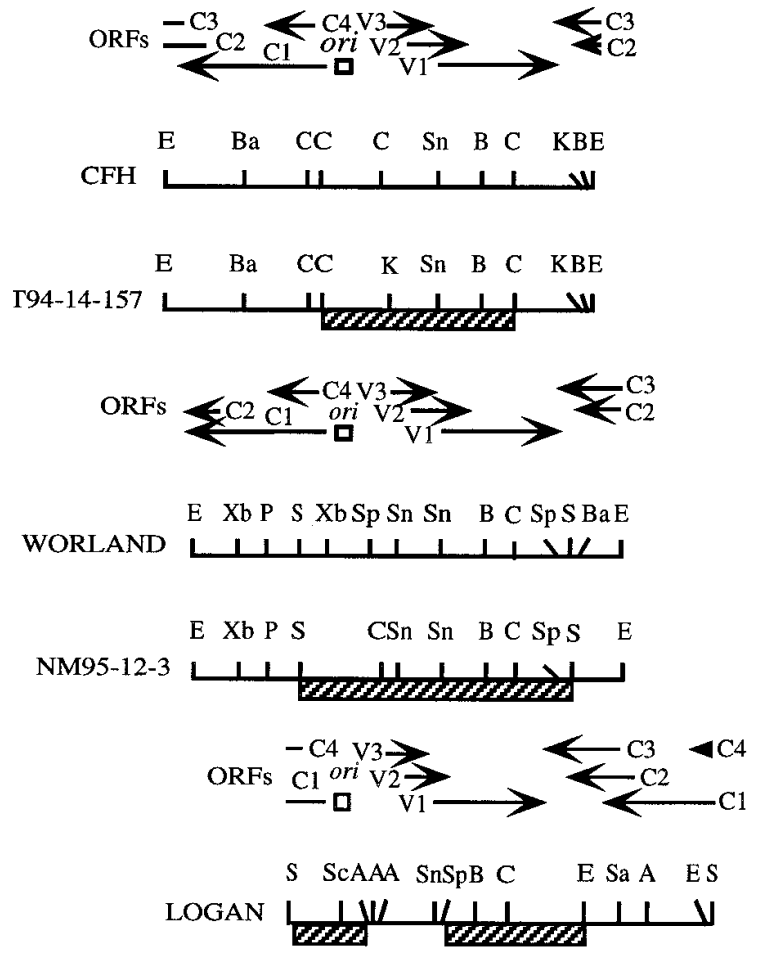

Fig. 1. Beet curly top virus (BCTV) helper virus and defective (D) DNA constructs. Presented are linear alignments of the cloned genomes of BCTVCFH, BCTV-Worland, and BCTV-Logan used as helper viruses. The location and polarity of open reading frames are indicated by arrows; the location of the origin of DNA replication (ori) is denoted as an open box. Sequences retained in the naturally occurring defective-interfering (DI) DNA of Logan or artificially constructed D DNAs of CFH (derived from pT94-14-157) or Worland (derived from pNM95-12-3) are denoted as striped boxes. Restriction sites present on each displayed genome are abbreviated as follows: ApaI (A), BamHI (Ba), BstXI (B), Csp45I (C), EcoRI (E), KpnI (K), PvuII (P), SalI (S), ScaI (Sc), SnaBI (Sn), SpeI (Sp), and $\mathrm{XbaI}(\mathrm{Xb})$.

\section{MATERIALS AND METHODS}

A naturally occurring DI DNA (LSG23) derived from the Logan genome was previously cloned as a 1,518-base pair (bp) Csp45I fragment (20). The LSG23 DI DNA is missing two regions of the Logan genome, truncating each of the seven viral-encoded ORFs. The LSG23 molecule has sequences of the ori that are also retained in D or DI DNAs derived from the closely related California isolate of BCTV (6). For simplicity, the LSG23 DI DNA is subsequently referred to in this paper as a D DNA. Artificial D DNAs of the Worland and $\mathrm{CFH}$ strains were constructed by subcloning DNA fragments from previously cloned $(14,17)$ genotypic variants of CFH (pT94-14-157) and Worland (pNM95-12-3). The CFH artificial D DNA was subcloned as a 1,250-bp Csp45I fragment in pGEM7Zf+ (pT94-14-157-D8), whereas the Worland artificial D DNA was subcloned as a 1,950-bp SalI fragment in pUC8 (pNM9512-3-D1). Both artificial D DNAs had extensive deletions in the C1 ORF encoding the Rep protein, yet retained ori sequences (Fig. 1). ori sequences for the two genotypic variants used to construct the artificial D DNAs were nearly identical to the prototype isolates of the respective strains (14; D. C. Stenger, unpublished data) and had cis-acting specificity elements that were identical to those of the respective prototype isolates (Fig. 2).

Tandemly repeated inserts for each artificial D DNA were constructed in the Agrobacterium tumefaciens binary vector pMON521 and introduced into A. tumefaciens as previously described for the tandemly repeated LSG23 DNA insert (13). Tandemly repeated constructs of the Logan, CFH, and Worland genomes in pMON521 $(16,19)$ served as inocula to deliver helper virus genomes to test their ability to trans-replicate BCTV D DNA constructs coagroinoculated to Nicotiana benthamiana Domin. leaf disk explants as previously described (2). Trans-replication of D DNAs by helper virus genomes was assessed by Southern blotting of total DNA samples extracted from $N$. benthamiana leaf disks using a genomelength Logan riboprobe of complementary sense transcribed from pCT10 (20).

\section{RESULTS AND DISCUSSION}

Southern blot analysis of total DNA samples extracted from $N$. benthamiana leaf disks (Fig. 3) indicated that helper virus progeny DNA was primarily single-stranded and of genome length. Some samples contained minor amounts of genome-length double-stranded replicative forms. Although all three strains of BCTV readily produce DI DNA, D DNA, or both de novo in systemically infected plants $(6,13)$, no less-than-genome-length virus-specific DNAs were detected in leaf disks agroinoculated only with helper virus genomes. This observation has been noted (2). As expected, the D DNA constructs were incapable of self-replication when helper virus inoculum was absent (Fig. 3). However, coinoculation of BCTV D DNA constructs with certain helper virus genomes led to amplification of less-than-genome-length DNA of the expected size (Fig. 3). Replicated D DNA progeny were also predominately single-stranded, although some double-stranded DNA products were observed (Fig.

\section{Consensus GGNGN $(3-5)$ GGNG \\ CFH GGTGCTTTGGGTG \\ WORLAND GGTGCTaTGGGaG \\ CAL/LOGAN GGaG-TaTtGGaG}

Fig. 2. Nucleotide sequences of the cis-acting strain-specific replication element of three beet curly top virus strains. The consensus sequence for curtoviruses and begomoviruses is shown at top. Lowercase letters indicate nucleotides that differ from the $\mathrm{CFH}$ sequence. 
3). As previously reported $(2,13)$, the Logan-derived LSG23 DNA was amplified only in the presence of a helper virus genome encoding the trans-acting replication specificity element of the Logan Rep protein. In contrast, the artificially constructed D DNAs of the CFH and Worland strains were amplified in the presence of either CFH or Worland as helper viruses. This indicates that cis- and transacting replication specificity elements of the $\mathrm{CFH}$ and Worland strains are compatible and functionally interchangeable.

A comparison of sequences in the cis-acting replication specificity element of the BCTV ori (Fig. 2) reveals that the 5'-proximal sequences are identical among the $\mathrm{CFH}$ and Worland strains, and overall, there are only two nucleotide substitutions. While all three strains have nucleotide substitutions within the central portion of the element, the 3'-proximal GGAG motif is identical among Cal/ Logan and Worland, but present as GGTG in CFH. In contrast, CFH and Worland share the $5^{\prime}$-proximal GGTG motif that is present as GGAG in Cal/Logan. These observations suggest that the 5 -proximal portion of the cis-acting element is critical to the recognition of the Rep protein and that some flexibility in sequence in the central and 3'-proximal portions of the element may be tolerated, although the entire element is required for Rep protein binding (4). This conclusion is consistent with the results of sitedirected mutagenesis experiments conducted on hybrid oris derived from the $\mathrm{CFH}$ and Logan genomes (3) or on those derived from begomovirus oris (5).

Alignment of Rep protein amino acid sequences encoded by the three strains of BCTV is presented in Figure 4. Numerous sequence differences appear within the region of the Rep protein previously demonstrated (2) to harbor the trans-acting replication specificity element (corresponding to Logan amino acid residues 3 to 89). Rep proteins of Logan and CFH differ at 35 positions within this region, and one or more of these differences should determine the strain specificity of the trans-acting element. A comparison of the Worland and Logan Rep protein amino acid sequences within this same region reveals 43 amino acid changes, whereas the Worland and $\mathrm{CFH}$ Rep proteins differ at 37 positions. The four-amino-acid-residue gap in the CFH Rep protein sequence (corresponding to Cal/ Logan and Worland Rep protein residues 3 to 6) would not account for the observed replication specificity phenotypes and may be considered as not part of the trans-acting specificity element. Despite the differences in Rep protein amino acid sequences that exist among BCTV strains, these comparisons suggest that the trans-acting replication specificity element resides in one or more of 12 amino acid residues that are identical; in two amino acid residues that are chemically similar among the CFH and Worland Rep proteins, yet different from the Logan Rep protein (Fig. 4); or in both. Confirmation of this hypothesis will require in-depth analysis of numerous site-directed mutants in which mutated Rep proteins encoded on one molecule may be tested for the ability to trans-replicate molecules bearing oris derived from heterologous BCTV strains.

The compatibility of $\mathrm{CFH}$ and Worland replication specificity elements has practical applications. Previous efforts to develop resistance based on transgenic plants bearing Logan (13) or California (7) DI DNAs resulted in improved resistance following challenge inoculation with the homologous helper virus. However, the protective effects of the Cal/Logan DI DNA transgenes were not extended to other geminiviruses (7) or even to heterologous strains of BCTV (13) used as challenge inocula. Furthermore, extensive sampling of BCTV populations in the western United States (14, 17) indicates that $\mathrm{CFH}$ and Worland are common in contemporary sugar beet production. The $\mathrm{Cal} / \mathrm{Logan}$ strain has been recovered only from isolates husbanded for extensive periods in laboratories $(11,15)$ or from populations maintained in cultivar evaluation nurseries (18). Therefore, transgenic DI DNA strategies designed to protect sugar beet from the Cal/Logan strain would be of questionable benefit if deployed in the field. However, because the CFH and Worland strains can trans-replicate heterologous D DNAs, transgenic sugar beets bearing a single DI DNA derived from either $\mathrm{CFH}$ or Worland should provide improved resistance to both strains of BCTV that are prevalent in the field today.

The taxonomy and nomenclature of BCTV strains has been problematic. All three BCTV strains share wide host range properties, are vectored by the same species of leafhopper, and cause, to varying severity, curly top symptoms in beet $(11,15)$. However, the nucleotide sequence identities (18) are well below the threshold of $90 \%$ identity to consider each strain as a separate virus species. Separate species designation for the Cal/Logan strain is further supported by the incompatibility of the replication specificity elements of the Logan genome with that of the $\mathrm{CFH}$ and Worland strains. However, the CFH and Worland strains do have replication specificity elements that are compatible with one another; therefore, should $\mathrm{CFH}$ and Worland be considered as strains of a single virus

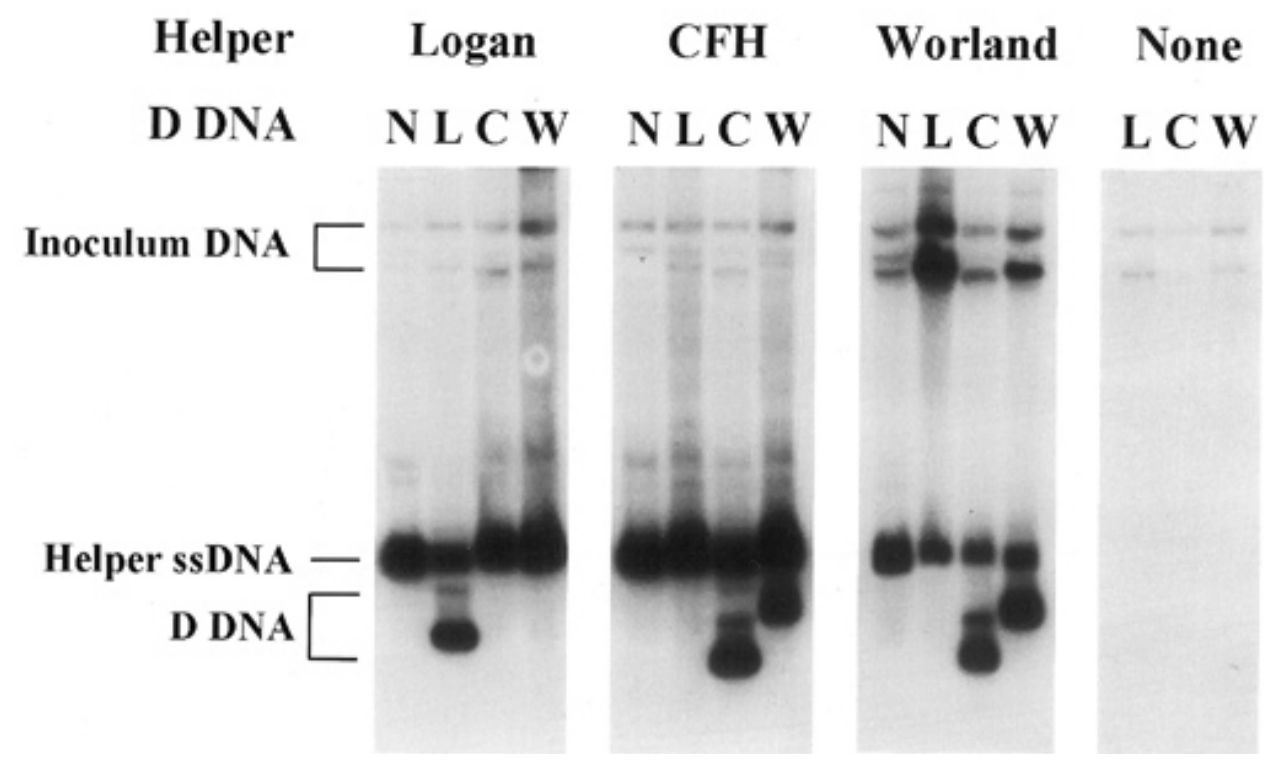

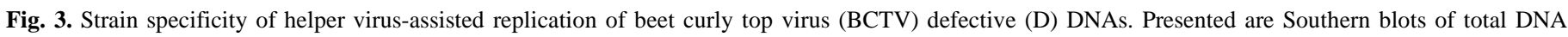

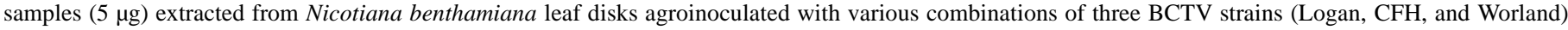

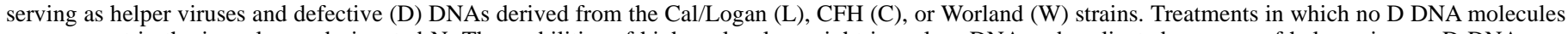

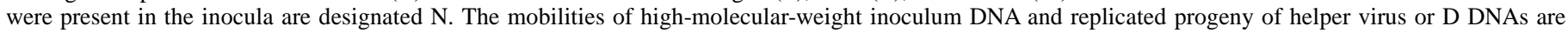
indicated at left. A full-length Logan riboprobe of complementary sense was used as a probe. 
even though the nucleotide identity of these two genomes is slightly less $(80.2 \%)$ than that between CFH and Logan (82.3\%)? In this case, sequence identity thresholds and trans-replication criteria established for geminivirus species conflict. However, $\mathrm{CFH}$ and Worland also differ in symptom severity on sugar beet (15) and latent period on $N$. benthamiana (13), representing pathogenic differences that also could be used as taxonomic characters to define separate species. It now seems appropriate that each of the three BCTV strains be designated as separate species. Therefore, I propose that California, Logan, and similar isolates represent a species that retains the name BCTV (and status as type species of the genus Curtovirus), because this was the first of the three to be sequenced (11). I further propose that $\mathrm{CFH}$, Iran, and similar isolates be designated as a new species of the genus Curtovirus, beet severe curly top virus (BSCTV), to reflect the short latent period and most severe symptoms produced by these isolates on $N$. benthamiana $(1,13,16)$. Finally, I propose that Worland and similar isolates also be designated as a new species of the genus Curtovirus, beet mild curly top virus (BMCTV), to reflect the mild symptoms produced in most cultivars of sugar beet (15). Adoption of this nomenclature would highlight the distinctions among the currently recognized strains of BCTV and would reduce confusion associated with geographical designations for virus species that do not convey the current or complete distribution of these viruses. Furthermore, this proposal would permit geographical point-of-origin to be retained as familiar identifiers of individual isolates (BCTV-Cal, BCTVLogan, BSCTV-CFH, BSCTV-Iran, and BMCTV-Worland), for which extensive literature exists.

\section{ACKNOWLEDGMENTS}

This material is based upon work supported by the Cooperative State Research Service, United States Department of Agriculture under agreement 93-37303-9384. Additional funds were provided by the Beet Sugar Development Foundation, Denver, CO.

\section{LITERATURE CITED}

1. Briddon, R. W., Stenger, D. C., Bedford, I. D., Stanley, J., Izadpanah, K., and Markham, P. G. 1998. Comparison of a beet curly top virus isolate originating from the old world with those from the new world. Eur. J. Plant Pathol. 104:77-84.

2. Choi, I.-R., and Stenger, D. C. 1995. Strain-specific determinants of beet curly top virus DNA replication. Virology 206:904-912.

3. Choi, I.-R., and Stenger, D. C. 1996. The strain-specific cis-acting element of beet curly top geminivirus DNA replication maps to the directly repeated motif of the ori. Virology 226:122-126.

4. Fontes, E. P. B., Eagle, P. A., Sipe, P. S., Luckow, V. A., and Hanley-

\begin{tabular}{|c|c|}
\hline & $\#$ \\
\hline Logan & MPPTKRFRIQAKNIFLTYPQCSLSKEEALEQIQGIQLSSNKKYIKIAREL \\
\hline $\mathrm{CFH}$ & MP----FYKKAKNFFLTYPQCSVTKEDALEQLLA INTPSNKKY IRICREL \\
\hline \multirow[t]{2}{*}{ Worland } & MPRSPSFQIKAKNIFLTYPRCSVIKEDALEILKNIPCPSDKLF IRVSQEK \\
\hline & 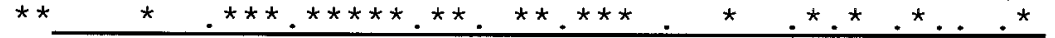 \\
\hline Logan & $\begin{array}{ccc}\# \# \# \# \text { \# } \# \text { A } & \# \text { \#A } \\
\text { HEDGQPHLHVLLQLEGKVQITNIRLFDLVSPTRSAHFHPNIQGAKSSSDV }\end{array}$ \\
\hline $\mathrm{CFH}$ & HENGEPHLHALIQFEGKVQIRNARYFDLQHRSTSKQFHCNIQGAKSSSDV \\
\hline Worland & 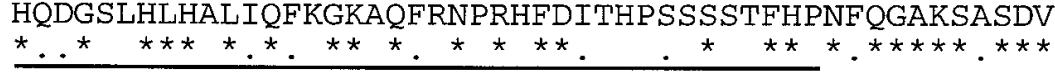 \\
\hline Logan & KSYVDKDGDTIEWGEFQIDGRSARGGQQTANDSYAKALNATSLEQALQIL \\
\hline $\mathrm{CFH}$ & KSYVSKDGDHIDWGEFQVDGRSARGGQQTANDAAAEALNAGNALEALQI I \\
\hline \multirow[t]{2}{*}{ Worland } & KQYIEKDGDYVDWGTFQVDGRSARGGQQTANDAAAEALNAGNAAEALQII I \\
\hline & 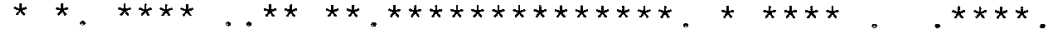 \\
\hline Logan & KEEQPKDYFLQHHNLLNNAQKIFQRAPDPWTPLF PLSSFTNVPEEMQEWA \\
\hline $\mathrm{CFH}$ & REKLPEKYIFQYHNLKPNLEAIFLPPPDLYQPPF PLSSFTRVPEIIQEWA \\
\hline \multirow[t]{2}{*}{ Worland } & REKLPEKYIFQYHNLKPNLEAIFLPPPDIYQPPF PQSSFTQVPEIVQEWA \\
\hline & 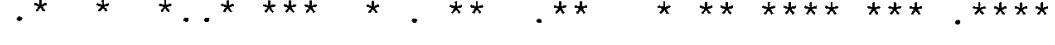 \\
\hline Logan & DAYFGVDAAARPLRYNSIIVEGDSRTGKTMWARSLGAHNYITGHLDFSLR \\
\hline $\mathrm{CFH}$ & DSYFGLDPAARPFRYNSLIIEGDSRTGKTMWARCLGPHNYITGHLDFSLK \\
\hline Worland & 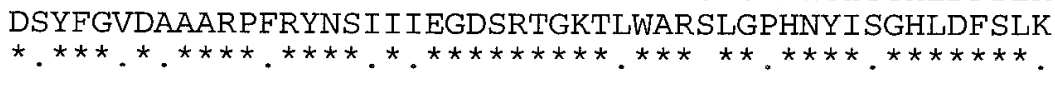 \\
\hline Logan & TYYDEVEYNVIDDVDPTYLKMKHWKHLIGAQKEWQTNLKYGKPRVIKGGI \\
\hline $\mathrm{CFH}$ & TYNDNVLYNVIDDVDPNYLKMKHWKHLIGAQREWQTNLKYGKPRVIKGGI \\
\hline Worland & 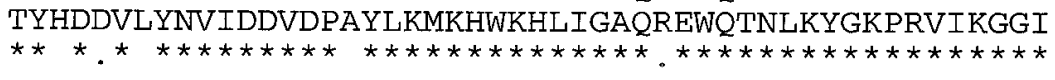 \\
\hline Logan & PCI ILCNPGPESSYQQFLEKPENEALKSWTLHNSTFCKLQGPLFNNQAAA \\
\hline $\mathrm{CFH}$ & PSIILCNPGEGSSYQDFLNKSENEALRSWTLQNSVFAKLTSPLFDNNQEA \\
\hline Worland & PSI ILCNPGDGSSYQDFLDKSENEALKSWTIQNSTFVKLSGPLFDKDTEA \\
\hline & 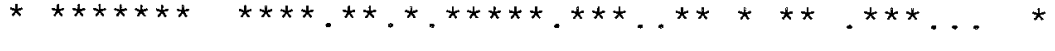 \\
\hline Logan & SSQGDSTL \\
\hline $\mathrm{CFH}$ & SSQDQTSL \\
\hline Worland & $\underset{* * \star}{S S Q G Q S P L}$ \\
\hline
\end{tabular}

Fig. 4. Alignment of replication initiator (Rep) protein amino acid sequences for three beet curly top virus strains. Amino acid seque nces are presented as a oneletter code. Asterisks $\left(^{*}\right)$ denote amino acid residues that are identical among all three strains, dots (.) denote amino acid residues that are chemically similar among all three strains. The region of the Rep protein previously demonstrated to contain the trans-acting replication specificity element is underlined. Within the region of the Rep protein containing the trans-acting replication specificity element are indicated the positions of 12 amino acid residues that are identical (\#) or 2 amino acid residues that are chemically similar $(\wedge)$ among the CFH and Worland Rep proteins, but different from the Log an Rep protein. 
Bowdoin, L. 1994. Interaction between a geminivirus replication protein and origin DNA is essential for viral replication. J. Biol. Chem. 269: 8459-8465.

5. Fontes, E. P. B., Gladfelter, H. J., Schaffer, R. L., Petty, I. T. D., and Hanley-Bowdoin, L. 1994. Geminivirus replication origins have a modular organization. Plant Cell 6:405-416.

6. Frischmuth, T., and Stanley, J. 1992. Characterization of a beet curly top virus subgenomic DNA localizes sequences required for replication. Virology 189:808-811.

7. Frischmuth, T., and Stanley, J. 1994. Beet curly top virus symptom amelioration in Nicotiana benthamiana transformed with a naturally occurring subgenomic DNA. Virology 200:826-830.

8. Hormuzdi, S. G., and Bisaro, D. M. 1993. Genetic analysis of beet curly top virus: Evidence for three virion sense genes involved in movement and regulation of single- and double-stranded DNA levels. Virology 193: 900-909.

9. Jupin, I., Hericourt, F., Benz, B., and Gronenborn, B. 1995. DNA replication specificity of TYLCV geminivirus is mediated by the amino-terminal 116 amino acids of the rep protein. FEBS (Fed. Eur. Biochem. Soc.) Lett. 362:116-120.

10. Sanz-Burgos, A. P., and Gutierrez, C. 1998. Organization of the cisacting element required for wheat dwarf geminivirus DNA replication and visualization of a Rep protein-DNA complex. Virology 243:119-129.

11. Stanley, J., Markham, P. G., Callis, R. J., and Pinner, M. S. 1986. The nucleotide sequence of an infectious clone of the geminivirus beet curly top virus. EMBO (Eur. Mol. Biol. Organ.) J. 5:1761-1767.
12. Stenger, D. C. 1994. Complete nucleotide sequence of the hypervirulent CFH strain of beet curly top virus. Mol. Plant-Microbe Interact. 7:154-157.

13. Stenger, D. C. 1994. Strain-specific mobilization and amplification of a transgenic defective-interfering DNA of the geminivirus beet curly top virus. Virology 203:397-402.

14. Stenger, D. C. 1995. Genotypic variability and the occurrence of less than genome-length viral DNA forms in a field population of beet curly top geminivirus. Phytopathology 85:1316-1322.

15. Stenger, D. C., Carbonaro, D., and Duffus, J. E. 1990. Genomic characterization of phenotypic variants of beet curly top virus. J. Gen. Virol. 71:2211-2215.

16. Stenger, D. C., Davis, K. R., and Bisaro, D. M. 1994. Recombinant beet curly top virus genomes exhibit both parental and novel pathogenic phenotypes. Virology 200:677-685.

17. Stenger, D. C., and McMahon, C. L. 1997. Genetic diversity of beet curly top virus populations in the western United States. Phytopathology 87:737-744.

18. Stenger, D. C., and Ostrow, K. M. 1996. Genetic complexity of a beet curly top virus population used to assess sugar beet cultivar response to infection. Phytopathology 86:1316-1322.

19. Stenger, D. C., Revington, G. N., Stevenson, M. C., and Bisaro, D. M. 1991. Replicational release of geminivirus genomes from tandemly repeated copies: Evidence for rolling-circle replication of a plant viral DNA. Proc. Natl. Acad. Sci. U.S.A. 88:8029-8033.

20. Stenger, D. C., Stevenson, M. C., Hormuzdi, S. G., and Bisaro, D. M. 1992. A number of subgenomic DNAs are produced following agroinoculation of plants with beet curly top virus. J. Gen. Virol. 73:237-242. 